\title{
Effect of static magnetic field on experimental dermal wound strength
}

\author{
Yahya Ekici, Cem Aydogan, Cenk Balcik, Nihan Haberal², Mahir Kirnap, Gokhan Moray, \\ Mehmet Haberal \\ Departments of General Surgery, 'Mechanical Engineering, and ${ }^{2}$ Pathology, Baskent University, Ankara, Turkey
}

Address for correspondence: Prof. Mehmet Haberal, Department of General Surgery, Baskent University, Cad. No: 77 Kat: 4 Bahcelievler, 06490 Ankara, Turkey. E-mail: rektorluk@baskent-ank.edu.tr

\section{ABSTRACT}

Context: An animal model. Aim: We sought to evaluate the effect of static magnetic fields on cutaneous wound healing. Materials and Methods: Male Wistar rats were used. Wounds were created on the backs of all rats. Forty of these animals (M group) had NeFeB magnets placed in contact with the incisions, either parallel $(\mathrm{Pa})$ and perpendicular $(\mathrm{Pr})$ to the incision. The other 40 animals (sham [S] group) had nonmagnetized NeFeB bars placed in the same directions as the implanted animals. Half of the animals in each group were killed and assessed for healing on postoperative day 7 and the other half on postoperative day 14. The following assessments were done: gross healing, mechanical strength, and histopathology. Statistical Analysis Used: Intergroup differences were compared by using the Mann-Whitney $U$ or $t$ test. Values for $P$ less than 0.05 were accepted as significant. Results and Conclusions: There were no differences between the magnetic and sham animals with respect to gross healing parameters. The mechanical strength was different between groups. On postoperative day 14, the MPr14 had significantly higher scores than the other groups. When static, high-power, magnetic fields are placed perpendicular to the wound, increased wound healing occurs in the skin of the experimental model.

\section{KEY WORDS}

Static electromagnetic field; wound healing; breaking strength; skin

\section{INTRODUCTION}

kin wound healing is a complex process and a continuing challenge in surgery. Despite recent advances, problems in wound healing cause significant morbidity and mortality. ${ }^{[1]}$ The complete process of wound healing is critical for the general well-being of the patient.

\begin{tabular}{|l|l|}
\hline \multicolumn{2}{|c|}{ Access this article online } \\
\hline Quick Response Code: & Website: \\
\hline & www.ijps.org \\
\cline { 2 - 2 } & Dol: \\
\hline
\end{tabular}

Several animal models have been used as an experimental basis to determine molecular and cellular mechanisms underlying and controlling an undisturbed healing process. Poffenbarger and Haberal reported that surgical or burn trauma produced an increase in serum nonresponsible insulinlike activity (NSILA) in an experimental model, and they showed its role in the tissue repair process. ${ }^{[2]}$

Experimental studies have focused on pulsed and static magnetic fields. Some of the findings show that lowfrequency pulsed electromagnetic fields accelerate skin wound healing and prevent necrosis. ${ }^{[3]}$ However, other studies have demonstrated that pulsed electromagnetic fields did not have a significant beneficial effect on wound healing. ${ }^{[4]}$ Use of static magnetic fields on 
different kinds of tissue defects can produce satisfying therapeutic effects. ${ }^{[5-8]}$ Research has shown that lowpower static magnetic fields increased the rate of wound healing, but no study to date has examined high-static magnetic fields effect on primary wound healing. ${ }^{[8]}$ In this study, we examined the effects of an externally applied electromagnetic field, a static magnetic field generated by a permanent $\mathrm{NeFeB}$ magnet, bidirectionally to skin wound healing and strength in rats.

Eighty male 4- to 5-month-old Wistar albino rats were used. The study was approved by the Baskent University Research Board and Ethics Committee. Animals were acclimatized to laboratory conditions for 1 week before the experiments, and were fed standard rat chow, and given free access to water throughout the study. Rats were housed individually in plastic cages, on a wooden bench. The distance between cages was $30 \mathrm{~cm}$, to prevent interaction between magnets.

\section{Study groups and procedures}

Eighty rats were divided into 2 groups: Those containing magnetic bar and those containing a nonmagnetic bar. Each group was further divided into 2 subgroups, depending on the route the bars took (parallel or perpendicular). Each subgroup was then further divided into 2 more subgroups according to the postoperative day on which it was examined (day 7 or day 14).

Forty of these 80 animals (M group) had NeFeB magnets (the product has been described in detail previously) placed near the incisions $(0.5 \mathrm{~cm}$ away), either parallel ( $\mathrm{Pa}$; the north seeking pole was positioned cranially) or perpendicular ( $\mathrm{Pr}$; the north seeking pole was positioned close to the midline) to the incision. (The magnetic field strength at the site was 390 to 420 gauss. ${ }^{[9]}$ ) To prevent inadvertent displacement, the bars were fixed to the skin by placing a 2-0 silk suture in a groove that the manufacturer had made at each pole of the bar. The other 40 animals (the sham $[S]$ group) had nonmagnetized $\mathrm{NeFeB}$ bars going in the same directions, dimensions, and weights implanted. Half of the animals in each group were killed by anesthetic overdose and assessed for healing parameters on postoperative day 7 (groups MPa7, MPr7, Pa7, and SPr7), and the other half was killed on postoperative day 14 (groups MPa14, MPr14, SPa14, and SPr14).

\section{Surgical procedure}

Animals were anesthetized with a combination of intraperitoneally administered $5 \mathrm{mg} / \mathrm{kg}$ xylazine
(Rompun, Bayer, Istanbul, Turkey) and $30 \mathrm{mg} / \mathrm{kg}$ ketamine hydrochloride (Ketalar, Parke-Davis, Istanbul, Turkey). The backs were shaved and prepared for using an aseptic technique. Three centimeter dorsal incisions were made through the epidermis and the dermis. The incision was made $3 \mathrm{~cm}$ away from the dorsal midline and parallel to the midline. All surgical procedures were performed by the same investigator. The wounds were then sutured with continuous 4-0 nylon (Ethicon, Inc, Somerville, NJ, USA). For postoperative analgesia, beginning on the day after the surgery, $0.02 \mathrm{mg} / \mathrm{kg}$ fentanyl citrate (Fentanyl; Abbott, Chicago, IL, USA) was administered subcutaneously, 2 times daily, for 3 days.

After each rat in the magnetized and nonmagnetized groups was killed, 4 types of evaluations were done.

The incision sutures were removed. Macroscopic examination included wound dehiscence, suture reaction, scar formation, infection, and abscess formation.

Ten animals in each group (MPa7, MPr7, SPa7, and SPr7) were killed on day 7, and the other groups (MPa14, MPr14, SPa14, and SPr14) were killed on day 14 after wounding. The dorsal pelt containing the healing scar was removed and cut at a right angle to the long axis of the wound into four $10-\mathrm{mm}$ wide strips. The strips were placed in a buffered Ringer's solution ( $\mathrm{pH} 7.4)$ and used within 30 minutes of recovering the pelt to assess breaking strength. Biomechanical tests were performed using an Instron Model 8874 (Buckinghamshire, UK) universal material testing system. Two sides of the strips were placed into custom-made mechanical grips. Grip length was selected as $10 \mathrm{~mm}$ on both sides and finegrade sandpapers were placed inside the grips to prevent slipping. Test length of the slips was selected at $30 \mathrm{~mm}$. Tests were performed at a constant speed of $1 \mathrm{~mm} /$ minute until breakage at the healing scar was observed. Force was measured with a 250-N load-cell attached to the testing frame, and test results were stored on a personal computer for further analysis.

Incisional skin biopsies of wounded mice were formalinfixed, paraffin-embedded, routinely processed, and stained with hematoxylin and eosin.

Hematoxylin and eosin-stained sections were analyzed for the following histologic features (as defined by Muehlberger and associates): ${ }^{[4]}$ epidermal alterations (including acanthosis and surface integrity), and dermal 
alterations (including granulation tissue and inflammatory response). Epidermal thickness was given by the number of layers, and surface integrity was decided whether focal erosions were present or absent. In addition, dermal alterations were scored semiquantitatively from 0 to 4 ( 0 , absent; 1 , minimal; 2 , mild; 3 , moderate; 4 , severe).

Data are presented as mean values \pm SEM. Pairwise differences were compared using the Mann-Whitney $U$ or $t$ test as appropriate. Values for $P$ less than 0.05 were accepted as statistically significant.

\section{RESULTS}

All animals recovered from surgery. They were killed at a designated time, and there were no deaths during the experiment. There was no wound dehiscence, superficial or subcutaneous infections, or hypertrophic scar formation determined in the groups at gross examination.

The mean \pm standard deviation tensile strength values for groups MPa7, MPr7, SPa7, and SPr7 were 1.5 \pm 0.3 , $2.8 \pm 1.5,1.4 \pm 0.5$, and $1.2 \pm 0.6(P=.001)$. After a pairwise comparison using the Mann-Whitney $U$ test, the mean skin wound tensile strength was found to be significantly higher in the MPr 7 group than in the other groups (MPa7 vs SPr7; $P=.04 ; \mathrm{MPa} 7$ vs MPr7; $P=.02 ; \mathrm{MPa} 7$ vsSPa7; $P=.1 ; \operatorname{SPr} 7$ vs MPr7; $P=.01 ; \operatorname{SPr} 7$ vs $\mathrm{SPa} 7 ; P=.5$; and MPr7 vs SPa $7 ; P=.007$ ) [Figure 1]. On the $14^{\text {th }}$ day, tensile strength values for groups MPa14, MPr14, Spa14, and SPr14 were $3.1 \pm 0.8,7 \pm 4.4$, $2.2 \pm 0.5$, and $2.2 \pm 1.3(P<.001$; Figure 1). The MPr14 group had significantly higher tensile strength than the other groups (MPa14 vs $\operatorname{SPr} 14 ; P=.1$; MPa14 vs MPr14; $P=.001$; MPa14 vsSPa14; $P=.004$; $\operatorname{SPr} 14$ vs MPr14; $P=.006 ;$ SPr14 vs SPa14; $P=.9$; and MPr14 vs SPa14; $P=.003$ ). Specifically, the tensile strength was statistically higher in group MPa14 than it was in groups SPr14 and SPa14 [Figure 1].

There was no wound dehiscence, hypertrophic scar, or abscess formation and superficial infection when gross healing was evaluated. Table 1 lists the results of light microscope evaluations. Epidermal thickness was nearly same as that seen in both magnetically treated and untreated wounds. The MPr7 and MPr14 groups had higher scores for epidermal thickness, but this difference was not statistically significant.

Granulation tissue scores are nonsignificantly high in the nonmagnetized groups at the first week.

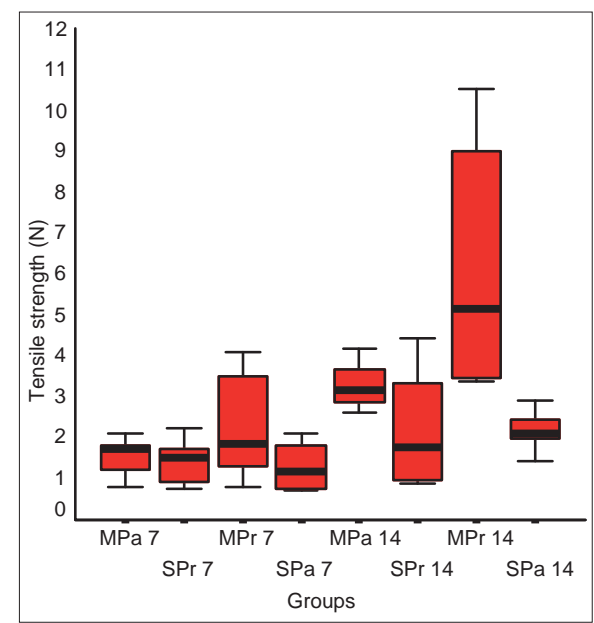

Figure 1: Breaking strength measurements of the groups in the first and second week

Granulation tissue scores are insignificantly higher in the MPr14 group when compared with the other groups. Wound biopsies sampled during the first and second weeks revealed no focal erosions. The MPr7 group had a lower inflammatory reaction than did the other groups, but were statistically insignificant. In the second week, all groups had the same values for inflammatory reaction

\section{DISCUSSION}

We used a bidirectional static magnetic field to the dermal incision, and found that exposure of a static magnetic field placed perpendicular to the incision increases the strength of cutaneous wounds that are closed primarily.

Two methods have been intensely investigated, and both positive and negative results have been reported. Pulsed electromagnetic field studies were differentiated according to intensity, vector, and exposure times of the magnetic field. Evidence has shown us that pulsed electromagnetic fields affect tissues as they undergo metabolic changes at the cellular level, especially during the healing process.

Basically, there are general problems when applying pulsed electromagnetic fields on dermal and other tissues. While experimentally used pulsed electromagnetic fields affect the whole body, it is difficult to investigate the net local effect of the magnetic field. Animals placed in cages have stable positions according to the vector of a pulsed electromagnetic field. To lessen the stress of test animals, exposure times must be limited. Application of static magnetic fields is simple, and it achieves a permanent 
Table 1: Breaking strength $(N)$ values in groups

\begin{tabular}{lcccc}
\hline Groups & $\boldsymbol{n}$ & Minimum $(\boldsymbol{N})$ & Maximum $(\boldsymbol{N})$ & Mean $\pm \mathbf{s d}$ \\
\hline MPa7 & 10 & 1 & 2.1 & $1.5 \pm 0.3$ \\
MPr7 & 10 & 1.27 & 5.47 & $2.8 \pm 1.5$ \\
SPa7 & 10 & 71 & 2.13 & $1.2 \pm 0.5$ \\
SPr7 & 10 & 16 & 2.25 & $1.4 \pm 0.5$ \\
MPa14 & 10 & 3.56 & 4.17 & $3.1 \pm 0.8$ \\
MPr14 & 10 & 1.44 & 16.68 & $7 \pm 0.4$ \\
Spa14 & 10 & 0.87 & 2.91 & $2.2 \pm 0.3$ \\
SPr14 & 10 & 4.45 & $2.1 \pm 1.2$ \\
\hline
\end{tabular}

magnetic effect and a permanent vectorial effect. Static magnetic fields are not related to electric energy, as no heat and electricity harms the tissues. At the same time, the magnetic force applied locally, but not to the whole body or surrounding tissues, have minimal exposure. This makes the static magnetic field a useful tool for the long term.

Medical applications of a magnetic field are generally reported as successful in musculoskeletal disease, but there is a lack of explanation about a specific molecular mechanism of magnetic field effect. ${ }^{[9]}$ Rosen and associates reported the magnetic field effect depends on diamagnetic anisotropic properties of membrane phospholipids in moderate-intensity magnetic field. ${ }^{[10]}$ According to Miyakoshi, static magnetic fields do not have a lethal effect related to magnetic strength. ${ }^{[1]]}$ The magnetic field of the earth has a strength of $0.5 \mathrm{G}$, which is equivalent to about $5 \times 10^{-5} \mathrm{~T}$. In this study, we used the magnetic bars that have permanent $4 \mathrm{mT}$ field at the poles and $0.4 \mathrm{mT}$ at the incision site.

Murayama and associates first reported exposure to $0.35 \mathrm{~T}$ placed sickled erythrocytes perpendicular to a magnetic field. ${ }^{[12]}$ Normal erythrocytes were placed parallel to an 8-T field in another study. ${ }^{[13]}$ Maximum 1.7 T-field affects bull sperm to an arranged perpendicular magnetic field. ${ }^{[14]}$ Forearm skin fibroblasts were placed parallel to 40-KG static magnetic field in vitro. ${ }^{[15]}$ Human glioblastoma and Schwann cells have been oriented perpendicular to a magnetic field. ${ }^{[16]}$ There are varying results about the orientation of cells depending on the cell type, intensity, and application time of a static magnetic field. Thus, in this study, we placed the magnets either parallel or perpendicular (according to the incision). Hoping that at least 1 of the vectorial effects of the static magnetic fields could enhance wound healing by aligning the cells perpendicular to incision. In vivo studies have shown that static magnetic fields promoted dermal healing in humans. ${ }^{[17]}$
High static magnetic field intensity decreased resting finger skin microcirculation in humans, ${ }^{[18]}$ moderate intensity influenced arteriolar diameters, and reduces edema formation in rats. ${ }^{[19]}$

More detailed investigations about the effect of electrical fields and pulsed electromagnetic fields have been done. When an electrical field is applied to wound edges locally, cells migrated to anode and this phenomenon is called electrotaxis or galvanotaxis. The cells changed the direction when the place of anode and the cathode changed. ${ }^{[20]}$ We do not know if there is magnetotaxis or magneto-rotational instability related to the magnetic field. In this study, we did not investigate the magnetorotational instability or histologically cellular alignment in the wound. Static magnetic fields have some effects on moving biological features, like blood or active cellular organelles. Magnetic force affects in a perpendicular angle to change the momentum of something by processing the direction to make a magnetic moment. This is known as the Larmor Precession.

There have been few studies on the effects of static magnetic fields at the cellular level. Buemi and associates showed that a $0.5-\mathrm{mT}$ static magnetic field exposure increases necrotic morphology, but this effect varies at the cell type ${ }^{\mid 21]}$ Cell cycle analysis did not differ when 1.5to 7-T was exposed for 24 hours. ${ }^{[22]}$ Static 0.2-T magnetic field exposed to gingival fibroblasts for 6 or 8 months did not show increased proliferation. ${ }^{\mid 23]}$ Five-hour exposure of 6-mT static magnetic field had no apoptotic or necrotic effect on HL-60 cells. ${ }^{[24,25]}$

In this study, we used the magnetic bars that had permanent $4 \mathrm{mT}$ fields at the poles and $0.4 \mathrm{mT}$ at the incision site. In rats with magnets, the epidermal thickness was insignificantly high in the first week, but not in the second week. There were no focal erosions in the groups in 2 weeks. We observed no statistical difference in granulation tissue and inflammation in the groups in both weeks. Static magnetic 
field treatment did not significantly affect the histologic wound-healing process.

We did not observe the arrangement of collagen and fibroblasts in histologic sections. These parameters possibly have limited value for wound healing assessment in this model.

Dermal strength is an important measure because it best describes the mechanical property of skin. We found that perpendicular static magnetic treatment of the wound increased the wound breaking strength when compared to the other groups in the first or second week; especially, when the parallel magnetic effect did not affect woundbreaking strength, as it did in the perpendicular group. The reason for this may be that the classic direction of cell migration in the wound is between the wounded edges or the reparative cells arranged perpendicularly to the static magnetic field. Maybe the direction of the static effect is the same direction of cell migration when the magnet is perpendicular to the wound, so rotation or alignment of the cells can be affected from the geomagnetic field. In conclusion, the static magnetic field we tested in this study increased the mechanical strength of an experimental rat in a dermal wound model.

\section{REFERENCES}

1. Peackok EE, Cohen IK. Wound healing. In: McCarthy JG, May JW, Littler JW, editors. Plasticsurgery. Philadelphia: WB Saunders; 1990. p. 161-85.

2. Poffenbarger PL, Haberal MA. Role of serum nonsuppressible insulin-like activity (NSILA) in wound healing. I. Influence of thyroparathyroidectomy on serum NSILA and wound healing in the rat. Surgery 1976;80:608-16.

3. Callaghan MJ, Chang El, Seiser N, Aarabi S, Ghali S, Kinnucan ER, et al. Pulsed electromagnetic fields accelerate normal and diabetic wound healing by increasing endogenous FGF-2 release. Plast Reconstr Surg 2008;121:130-41.

4. MuehlbergerT, MoresiJM, Schwarze H, Hristopoulos G, LaengerF, Wong $L$. The effect of topical tretinoin on tissue strength and skin componentsinamurineincisionalwoundmodel.JAmAcadDermatol 2005;52:583-8.

5. Darendeliler MA, Darendeliler A, Sinclair PM. Effects of static magnetic and pulsed electromagnetic fields on bone healing. Int J Adult Orthodon Orthognath Surg 1997;12:43-53.

6. Bertolino G, de Freitas Braga A, de Oliveira Lima do Couto Rosa K, de Brito Junior LC, de Araujo JE. Macroscopic and histological effects of magnetic field exposition in the process of tissue reparation in Wistar rats. Arch Dermatol Res 2006;298:121-6.

7. Kelleher MO, Al-Abri RK, Lenihan DV, Glasby MA. Use of a static magnetic field to promote recovery after peripheral nerve injury. $\mathrm{J}$ Neurosurg 2006;105:610-5.

8. Henry SL, Concannon MJ, Yee GJ. The effect of magnetic fields on wound healing: experimental study and review of the literature. Eplasty 2008;8:e40.

9. Aydin N, Bezer M. The effect of an intramedullary implant with a static magnetic field on the healing of the osteotomised rabbit femur. Int Orthop 2011;35:135-41.

10. Rosen AD. Effect of a $125 \mathrm{mT}$ static magnetic field on the kinetics of voltage activated $\mathrm{Na}+$ channels in $\mathrm{GH} 3$ cells. Bioelectromagnetics 2003;24:517-23.

11. Miyakoshi J. Effects of static magnetic fields at the cellular level. Prog Biophys Mol Biol 2005;87:213-23.

12. Murayama M. Orientation of sickled erythrocytes in a magnetic field. Nature 1965;206:420-2.

13. Higashi T, Yamagishi A, Takeuchi T, Kawaguchi N, Sagawa S, Onishi $\mathrm{S}$, et al. Orientation of erythrocytes in a strong static magnetic field. Blood 1993;82:1328-34.

14. Emura R, Takeuchi T, Nakaoka Y, Higashi T. Analysis of anisotropic diamagnetic susceptibility of a bull sperm. Bioelectromagnetics 2003;24:347-55.

15. Guido S, Tranquillo RT. A methodology for the systematic and quantitative study of cell contact guidance in oriented collagen gels. Correlation of fibroblast orientation and gel birefringence. J Cell Sci 1993;105(Pt 2):317-31.

16. Hirose $\mathrm{H}$, Nakahara $\mathrm{T}$, Miyakoshi J. Orientation of human glioblastoma cells embedded in type I collagen, caused by exposure to a $10 \mathrm{~T}$ static magnetic field. Neurosci Lett 2003;338:88-90.

17. Man $\mathrm{D}$, Man $\mathrm{B}$, Plosker $\mathrm{H}$. The influence of permanent magnetic field therapy on wound healing in suction lipectomy patients: a double-blind study. Plast Reconstr Surg 1999;104:2261-6; discussion 2267-8.

18. Mayrovitz HN, Groseclose EE. Effects of a static magnetic field of either polarity on skin microcirculation. Microvasc Res 2005;69:24-7.

19. Morris CE, Skalak TC. Acute exposure to a moderate strength static magnetic field reduces edema formation in rats. Am J Physiol Heart Circ Physiol 2008;294:H50-7.

20. Zhao M, Pu J, Forrester JV, McCaig CD. Membrane lipids, EGF receptors, and intracellular signals colocalize and are polarized in epithelial cells moving directionally in a physiological electric field. FASEB J 2002;16:857-9.

21. Buemi M, Marino D, Di Pasquale G, Floccari F, Senatore M, Aloisi C, et al. Cell proliferation/cell death balance in renal cell cultures after exposure to a static magnetic field. Nephron 2001;87:269-73.

22. Schiffer IB, Schreiber WG, Graf R, Schreiber EM, Jung D, Rose DM, et al. No influence of magnetic fields on cell cycle progression using conditions relevant for patients during MRI. Bioelectromagnetics 2003;24:241-50.

23. Yamaguchi $H$, Hosokawa $K$, Soda A, Miyamoto $H$, Kinouchi $Y$. Effects of seven months' exposure to a static $0.2 \mathrm{~T}$ magnetic field on growth and glycolytic activity of human gingival fibroblasts. Biochim Biophys Acta 1993;1156:302-6.

24. Teodori L, Grabarek J, Smolewski P, Ghibelli L, Bergamaschi A, De Nicola $M$, et al. Exposure of cells to static magnetic field accelerates loss of integrity of plasma membrane during apoptosis. Cytometry 2002;49:113-8.

25. Flipo D, Fournier M, Benquet C, Roux P, Le Boulaire C, Pinsky C, et al. Increased apoptosis, changes in intracellular $\mathrm{Ca} 2+$, and functional alterations in lymphocytes and macrophages after in vitro exposure to static magnetic field. J Toxicol Environ Health $\mathrm{A}$ 1998;54:63-76.

How to cite this article: Ekici Y, Aydogan C, Balcik C, Haberal N, Kirnap M, Moray G, et al. Effect of static magnetic field on experimental dermal wound strength. Indian J Plast Surg 2012;45:215-9.

Source of Support: Nil, Conflict of Interest: None declared. 